Revue musicale OICRM

\title{
Germaine Tillion
}

\section{Credo du « solidus » et sympathie à la lettre}

\section{Djemaa Maazouzi}

Volume 3, numéro 2, 2016

Mémoire musicale et résistance. Autour du Verfügbar aux Enfers de Germaine Tillion

URI : https://id.erudit.org/iderudit/1060105ar

DOI : https://doi.org/10.7202/1060105ar

Aller au sommaire du numéro

Éditeur(s)

OICRM

ISSN

2368-7061 (numérique)

Découvrir la revue

Citer cet article

Maazouzi, D. (2016). Germaine Tillion : credo du « solidus » et sympathie à la lettre. Revue musicale OICRM, 3(2), 22-37. https://doi.org/10.7202/1060105ar
Résumé de l'article

Face à l'adversité - misère, totalitarisme, violence -, Germaine Tillion a montré que l'expérience individuelle peut devenir connaissance et la connaissance partagée se muer en action groupale. Toute sa trajectoire tient dans cette éthique qui est aussi mise en pratique solidaire liant étroitement observation, dialogue et réflexivité. Ce credo anime la vie de Tillion en amont et en aval de sa déportation à Ravensbrück en un " double apprentissage » qui allie l'étude et l'expérience sans les dissocier ni les hiérarchiser quant à leur apport commun de connaissances. Ce credo traverse de manière performative l'œuvre collective du Verfügbar aux Enfers. 


\title{
Germaine Tillion. Credo du « solidus " et sympathie à la lettre

\author{
Djemaa Maazouzi
}

\begin{abstract}
Résumé
Face à l'adversité - misère, totalitarisme, violence -, Germaine Tillion a montré que l'expérience individuelle peut devenir connaissance et la connaissance partagée se muer en action groupale. Toute sa trajectoire tient dans cette éthique qui est aussi mise en pratique solidaire liant étroitement observation, dialogue et réflexivité. Ce credo anime la vie de Tillion en amont et en aval de sa déportation à Ravensbrück en un "double apprentissage " qui allie l'étude et l'expérience sans les dissocier ni les hiérarchiser quant à leur apport commun de connaissances. Ce credo traverse de manière performative l'œuvre collective du Verfügbar aux Enfers.
\end{abstract}

Mots clés : care ; engagement ; ironie ; solidarité ; Germaine Tillion.

\begin{abstract}
In the face of adversity-misery, totalitarianism and violence-, Germaine Tillion showed that individual experience can become knowledge and that shared knowledge can mutate into group action. The entire trajectory of her life was embodied in an ethos reflecting these principles and converting into action a kind of solidarity that combined observation, dialogue and the ability to reflect. These principles were exemplified in Tillion's life both before and after her deportation to Ravensbrück in a form of "double learning" in which study and experience were intimately connectedand not differentiated qualitatively - in terms of their common contribution to knowledge. The entire collective work of Le Verfügbar aux Enfers ("The Campworker goes to Hell") is permeated with these principles in a performative manner.
\end{abstract}

Keywords: care; engagement; irony; solidarity; Germaine Tillion.

Dans son nécessaire ouvrage Mémoire du mal, tentation du bien. Enquête sur le siècle publié en 2000 et dans lequel il choisit de faire le portrait de certaines figures exceptionnelles de l'engagement du $\mathrm{xx}^{\mathrm{e}}$ siècle, Tzvetan Todorov clôt sa présentation de Germaine Tillion par cette image :

[A]ujourd'hui vieille dame, [elle] est l'un des être publics les plus lumineux qu'il nous soit donné de connaître dans ce siècle des ténèbres. On se prend à rêver qu'elle saisisse le genre humain par le col de sa chemise, qu'elle le secoue 
un peu et lui demande, du haut de son expérience : "Avez-vous compris ce que j'ai dit? » Et que celui-ci réponde, penaud : «Oui, m'dame... » (Todorov 2000, p. 359).

Todorov opte ainsi pour l'exemplification de la scène narrée par Tillion ${ }^{1}$ et Yacef Saadi (Saadi [1984]1997) lors de sa rencontre avec les combattants indépendantistes FLN en pleine bataille d'Alger. À la fin de plusieurs heures de discussions ${ }^{2}$ sur l'oppression, la répression, les violences coloniales et la riposte anticoloniale à l'issue desquelles Tillion fait promettre à Saadi de ne plus commettre d'attentats contre les civils alors qu'elle s'engage à faire arrêter les exécutions capitales d'Algériens, la vieille dame secoue par son col l'ex-voyou de la Casbah, Ali Lapointe. Elle fait promettre au jeune homme - devenu, après sa mort survenue quelques semaines plus tard, martyr de la révolution algérienne - d'arrêter les attentats à la bombe dans Alger.

En retour de cette promesse tenue des indépendantistes algériens, Tillion n'obtiendra pas l'arrêt des exécutions capitales, mais elle n'aura de cesse de se démener pour "sauver des têtes", littéralement, du couperet de la guillotine ainsi que pour extirper des mains de leurs tortionnaires nombre de détenues et détenus algériens et français ${ }^{3}$. Si l'on rajoute à l'image d'Épinal retenue par Todorov les effets de cette rencontre sur les protagonistes, l'action de la vieille dame n'en demeure pas moins édifiante. Le genre humain est capable d'atrocités et ces atrocités sont parfois commises en réponse à d'autres atrocités : les indépendantistes algériens ont retenu la leçon de Tillion et Tillion, elle, a compris la raison indépendantiste. Cette rencontre et ce dialogue lui ont fait réexaminer ses positions à l'aune de ses propres combats et expériences de détention, torture, humiliations, souffrances. Déjà aimée et respectée en Algérie pour son engagement social et humaniste, la patriote, gaulliste, ni anticolonialiste, ni pro-indépendantiste Germaine Tillion deviendra une « figure de l'émancipation algérienne »

1 Nancy Wood (2003), dans le chapitre de son ouvrage consacré à la torture durant la guerre d'Algérie, puise son information sur cette rencontre dans la déposition que Germaine Tillion devait envoyer à un tribunal d'Alger avant d'apparaître en tant que témoin de moralité pour Yacef Saadi pendant son procès. Cette déposition a été publiée dans L'Express du 28 août 1958. Cette version, selon Wood, est identique à celle qui se trouve dans le chapitre "Témoignage pour un homme condamné à mort " dans Les ennemis complémentaires (Tillion 1960).

2 On retrouvera quelques bribes de ce dialogue à plusieurs décennies d'intervalle sur France Culture, le 14 août 1996. Ce troisième épisode du documentaire de Patrice Gélinet intitulé « La Guerre d'Algérie, vingt-cinq ans après : l'engrenage 1956 », diffusé dans l'émission "L'histoire immédiate » est retranscrit dans son entièreté sur le site Fabrique de sens : http://www.fabriquedesens.net/La-guerre-d-Algerie-vingtcinq-ans, 458 (consulté le 15 janvier 2016).

Cette rencontre Tillion-Saadi est au centre du Dialogue adapté et mis en scène par Jean Quercy d'après Les ennemis complémentaires de Germaine Tillion (2005b) avec Sophie Million, Fatima El Hassouni, Niddal El Mellouhi et Éric Auvray. Créé en mars 2011 au Lavoir Moderne Parisien, le spectacle est repris au Musée Jean Moulin puis à la Crypte Saint Sulpice en 2012 et 2013. Il est présenté à l'occasion de l'entrée au Panthéon de Germaine Tillion par la compagnie Théâtre Averse Tabarmukk au Musée du Général Leclerc de Hauteclocque et de la Libération de Paris - Musée Jean Moulin, avec le parrainage de Stéphane Hessel et de la Ligue des Droits de l'Homme.

3 Voir Wood 2003, "Chapitre 5. La torture en Algérie. L'épreuve cruciale », p. 196-239. Wood y détaille notamment les diverses lettres envoyées par Germaine Tillion à De Gaulle entre 1958 et 1961. 
(Kelle [2013]2014) en reconnaissant, dans la lutte pour l'indépendance de l'Algérie, la résistance légitime à l'occupation française. Cet apprentissage de la résistance à l'occupation allemande et de la déportation n'a pas été nécessairement partagé, tant s'en faut ${ }^{4}$. Lors de la guerre d'Algérie, parfois, les victimes du passé nazi y sont même devenues les bourreaux du présent colonial français ${ }^{5}$ (Vidal-Naquet 1972).

\section{ENGAGEMENT ET DIALOGUE}

Germaine Tillion est un personnage dont l'exemple peut inspirer, car son exceptionnalité ne réside pas dans une somme de vertus innées qu'elle aurait égrenée en œuvre utile au fil de sa vie. C'est plutôt l'existence qui, par ses circonstances, ses accidents plus ou moins heureux ou tragiques, a conduit l'ethnologue à s'adapter et à adopter des manières d'agir et de penser suffisamment souples pour être questionnées, remaniées, remises en question, améliorées. Agir chez Tillion c'est réagir à une situation en étant forte d'une pensée aguerrie dans l'expérience d'une action précédente. Même systématisée simplement de cette manière, l'opération n'est bien entendu jamais coupée de ses contextes personnel, professionnel, politique et, plus largement, sociohistorique. Somme toute, comme l'ont montré plusieurs chercheurs ${ }^{6}$ (Vidal-Naquet 1995, 2007 ; Wood 2003 ; Reid 2004, 2007 ; Kelle [2013]2014), le fait remarquable chez Tillion est qu'elle a traversé le $\mathrm{xx}^{\mathrm{e}}$ siècle en tentant de modifier des situations révoltantes, injustes, iniques, non seulement tout en étant elle-même transformée par ses combats, mais en prenant acte des changements opérés en elle.

Femme ethnologue (Bromberger et Todorov 2002) dans l'Algérie coloniale des années 1930, résistante dans le Réseau du Musée de l'homme sous l'occupation allemande dès 1940, prisonnière détenue à Fresnes en 1942, déportée en 1943 dans le camp de femmes de Ravensbrück, Germaine Tillion ${ }^{7}$ devient historienne des camps de concentration à la fin de la Seconde Guerre mondiale. Engagée en 1951 aux côtés de David Rousset, créateur de la Commission internationale contre le régime concentrationnaire, elle retourne en Algérie en 1954 à la demande du gouvernement français

\footnotetext{
4 Mais à chaque fois que cela l'a été, cela a pu être le fait d'une "mémoire exemplaire (Todorov 1995) ou comme l'a conceptualisée Rothberg, " une mémoire multidirectionnelle ». Voir l'étude de Michael Rothberg (2009), Multidirectional Memory. Remembering the Holocaust in the Age of Decolonization, qui montre, à travers l'analyse de divers films et textes, la façon dont l'événement de la guerre d'Algérie a réveillé la mémoire du génocide juif en France.

5 Voir la conversation entre René Vautier et Germaine Tillion en 1985 sur l'engagement durant la guerre d'Algérie directement lié à la Seconde Guerre mondiale (Vautier 2013) : dialogue inédit où est évoquée la rencontre avec Yacef Saadi.

6 On retrouvera ces apprentissages de parcours analysés finement en tenant compte notamment : pour Vidal-Naquet $(1995,2007)$, de l'évolution de la compréhension des mécanismes du système concentrationnaire ; pour Wood (2003), des limites de "l'humanisme ethnographique » ; pour Reid (2004, 2007), du colonialisme et des rapports à la question de l'indépendance algérienne ; ou encore pour Kelle ([2013]2014), de la conception des rapports entre Algériens et Français.

7 On retrouvera consignées de manière précise et concise, sur le site qui lui est consacré, les informations essentielles de sa biographie : http://www.germaine-tillion.org/a-la-rencontre-de-germainetillion/biographie/1940-1954-resistance-et-deportation/ (consulté le 15 janvier 2016).
} 
qui tente alors de contrer les débuts d'une guerre anticoloniale. Elle y met en place les bases des centres sociaux ${ }^{8}$ (Forget 2013, Ould Aoudia 2013) destinés à diminuer la misère des " indigènes ${ }^{9}$ " (enfants et adultes) en permettant leur accès à l'éducation (Tillion 1960). En 1957, tout en condamnant les attentats des indépendantistes, elle s'engage contre la torture perpétrée par l'armée française et mobilise ses efforts contre les exécutions capitales en Algérie.

Active militante pour le droit à l'instruction des détenus dans les prisons françaises, elle publie, au sortir de la guerre d'Algérie, deux ouvrages sur la situation économique et sociale algérienne ${ }^{10}$. En 1966, elle fait paraître son étude sur les structures familiales en Méditerranée ${ }^{11}$ reprenant des grandes lignes de ses premiers travaux d'ethnologue dans les Aurès des années $1930^{12}$. Au début des années 1970, alors que le racisme anti-arabe est à son acmé en France, Germaine Tillion rejoint le front antiraciste hétérogène $^{13}$ qui voit le jour. Elle agit ainsi comme ceux qui s'étaient déjà élevés, au nom des principes qui les avaient fait combattre le nazisme, contre la torture durant la guerre d'Algérie et contre le colonialisme. En 1973, la première tâche à laquelle elle s'était consacrée une fois libérée du camp, son enquête sur Ravensbrück rédigée en 1946 (Tillion et al. 1946), est remaniée et substantiellement enrichie par une patiente et méticuleuse recherche documentaire qui couvre des investigations menées sur 30 années (Tillion 1973). En 1988, ce travail est repris et fondu dans une troisième édition du livre éponyme, Ravensbrück III, transformant progressivement un travail de mémoire des camps en une écriture de l'histoire du système concentrationnaire (Tillion 1988) ${ }^{14}$. Tout au long des années 1970, 1980, 1990 et jusqu'à son décès, Germaine Tillion ne cessera d'œuvrer dans des missions en Algérie, au Maroc, en Mauritanie, en Égypte, au Mali, au Niger comme chercheuse du cNRs ou engagée humanitaire de l'OMS et de l'oNU. Défenderesse active de l'amélioration de la situation des femmes immigrées, militante pour les droits des minorités ou encore contre l'esclavage moderne, le dialogue qu'elle entame avec les jeunes générations, hommes

8 Pour Nancy Wood, «l'histoire complète des centres sociaux reste à écrire. Il faut noter toutefois que Germaine Tillion lança ce programme de réforme ambitieux à partir d'un "manifeste" qui n'envisageait rien de moins que la scolarisation complète de l'Algérie. Elle était convaincue que seul un effort concerté allié à un investissement massif de ressources permettant l'éducation rapide de l'ensemble de la population musulmane et l'amélioration de leur niveau de vie pourrait aider les Algériens à affronter les exigences de la modernité qui arrivait dans leur pays. » (Wood 2003, p. 16)

9 Germaine Tillion, dans L'Algérie en 1957, qualifie de "clochardisation » le phénomène de la paupérisation des paysans sans terre et sans avenir (Tillion 1957, p. 27).

10 L'Afrique bascule vers l'avenir et Les ennemis complémentaires (Tillion 1960).

11 Le harem et les cousins (Tillion 1966). Voir le texte de Christian Bromberger (2009) qui démontre l'apport avant-gardiste et majeur de cette étude sur la spécificité des structures matrimoniales dans le monde méditerranéen.

12 À propos de ces travaux, voir notamment : http://www.germaine-tillion.org/a-la-rencontre-de-germaine-tillion/biographie/1934-1940-ethnologue-dans-les-aures/ (consulté le 29 février 2016).

13 Sur ce climat « anti-arabe » et la mobilisation antiraciste, lire Gastaut 2000.

14 Sur les trois versions de Ravensbrück, voir également l'article de Marie-Hélène Benoit-Otis et Philippe Despoix dans le présent numéro. 
et femmes contribue à l'édification de sa personnalité d'icône de la générosité et de l'engagement pour les causes justes aux côtés des plus défavorisés et fragilisés de la société.

\section{RÉFLEXIVITÉ ET SINCÉRITÉ}

Ainsi, pour qui examine le parcours de Germaine Tillion à travers ses actions, sa parole, ses écrits ${ }^{15}$, il n'échappe pas que l'ethnologue a traversé le $\mathrm{xx}^{\mathrm{e}}$ siècle en s'occupant de politique. Elle s'est en effet mêlée des affaires de la cité à des moments de tourments coloniaux, postcoloniaux, dans des contextes de guerres et de résistances. Elle a agi en toute liberté ou incarcérée ; dans le camp des persécutés, des opprimés ; à partir de celui de la force et de l'hégémonie, dans le cercle de ceux qui dominent. Tillion a éprouvé de véritables outils de distanciation qui servent non pas à demeurer indifférent en accusant la fatalité, pas plus à s'abstraire du contingent en théorisant et encore moins à perdre la priorité du souci immédiat - impératif - de la préservation de la vie humaine. Bien au contraire, chez Tillion, se distancier c'est rassembler les ressources de l'expérience, de la connaissance et de la pensée pour observer la situation, tenter d'en analyser des mécanismes.

Chez Tillion, l'expérience est retenue comme subjectivité d'un savoir. La connaissance est conçue comme objectivation d'une expérience. Et l'action est engagée comme révélation de soi dans la relation à l'autre face à l'arbitraire. Voilà, au-delà de la leçon de vie qu'offre son parcours exemplaire (Todorov 2000, 2007), les constantes d'un faire face à la vie qui tire remarquablement ses leçons des moments singuliers l'ayant ponctuée. Si " comprendre la nature humaine " (Todovov 2000, p. 360 ; Todorov 1991, p. 95 et 143-144) a été la visée de l'existence de Germaine Tillion (Tillion 1973, p. 186) - « comprendre » synonyme d' exister» (Tillion 2001, p. 37) -, cette trajectoire possède des principes forts : la recherche de la connaissance de l'autre afin de mieux se connaître soi-même ; l'impératif d'agir pour ne pas seulement subir et celui de réfléchir pour se donner les moyens d'agir ; l'utilisation de l'expérience comme outil de connaissance scientifique ; l'intégration du sujet au cœur du savoir ; la transformation du sujet en objet de savoir ; l'utilisation du savoir de l'expérience pour agir à nouveau. «Plusieurs fois dans ma vie, rappelle Tillion, j'ai eu l'occasion de créer de toutes pièces des systèmes d'investigation sociale pour des groupes humains entre lesquels nul lien n'existe : sociétés stables de paysans ou de nomades africains ou sociétés instables créées par l'événement et dissoutes avec lui » (Tillion 2009, p. 255).

15 Comme le souligne Christian Bromberger (2009, p. 12): "À chaque étape de ce parcours correspond un livre, complété et remanié, par souci d'exactitude, à chaque réédition : Ravensbrück (1946, 1973, 1988), L'Algérie en 1957 (dont la version augmentée est devenue L'Afrique bascule vers l'avenir, 1960, 1999), Les ennemis complémentaires $(1960,2005 b)$, Le harem et les cousins $(1966,1982)$. Quant à Il était une fois l'ethnographie (2000), c'est le compte rendu d'une partie des enquêtes que Germaine Tillion mena dans l'Aurès dans les années 1930 (le reste de ses notes et de ses manuscrits disparut à Ravensbrück). » 
La trajectoire de Germaine Tillion conjugue des principes éthiques fondés sur l'engagement moral « pour le vrai, le juste» (Tillion 1998, p. 3), sur l'attention à l'autre, sur le soin de la relation à l'autre comme part d'une meilleure compréhension de soi, sur la solidarité, la fraternité, l'amitié. Cette conscience de sa propre expérience des êtres, des choses, des événements et sa réutilisation au présent incorpore distanciation et dialogue et fonde, en précurseur original - si on prend comme seul exemple le reflexive turn de l'anthropologie comme discipline (Bromberger 2009) - une réflexivité opératoire et éminemment féconde. Ces principes éthiques ont à chaque fois été éprouvés dans une conception de l'ethnographie - définie par Tillion comme " un humanisme dans le sens où deux fils se croisent en un constant aller vers l'autre et retour vers soi » (Bouchet et Anthonioz 1974) - qui place en son cœur (prenons cette expression comme littérale générosité pour insister sur le souci de l'autre) la bonne foi " comme remise en question continuelle de soi " (ibid.). Cette sincérité ${ }^{16}$ dans l'effort de demeurer soi-même, c'est-à-dire à chaque fois dans la conscience de son dire et de son faire " avec » l'autre, est constitutive de la distanciation mise en ouvre, en situation ${ }^{17}$. Elle aurait à voir avec

[un] souci de disposer en pleine propriété de soi comme être sincère. [La sincérité] pourrait alors se définir comme l'effort pour renoncer à une représentation une et figée de soi, comme l'effort pour se voir dans les mots, dans ce qui fait le lien relationnel, à savoir la parole adressée. (Chiantaretto 2005, p. 105)

Quant à l'action qui est selon Tillion une contrainte au choix, " au choix dans un sens ou un autre " (Bouchet et Anthonioz 1974), elle révèle l'individu face à l'ordinaire, face à l'extrême, face à une conduite morale où " ce qui compte entre les individus, ce n'est pas moi ou l'autre, mais la relation entre les deux [car] seuls les tissus de relations importent vraiment » (Reid 2007, p. 151).

\section{LA LEÇON D’APPRENTISSAGE DU VERFÜGBAR}

Aussi, face à l'adversité - misère, totalitarisme, violence comme les a si bien cernés Todorov -, Tillion a montré que l'expérience individuelle peut devenir connaissance et la connaissance partagée, se muer en action groupale. Toute sa trajectoire tient dans

\footnotetext{
16 On pourrait considérer d'ailleurs que dans le cas de Germaine Tillion, cette sincérité est celle-là même que définit Jean-François Chiantaretto (2005, p. 103), pour lequel il n’y a de sincérité « que dans le projet d'une parole adressée dans le face-à-face avec l'altérité d'autrui - avec autrui en tant qu'il est à la fois irréductible à mes représentations et le lieu d'une attente au moins potentielle, me donnant accès, à ce titre, à ma propre altérité. »

17 De cette sorte de situation particulière que définit Derrida (1996, p. 48-49) et qui est la sienne lorsqu'il se remémore l'Algérie sous Vichy : "Il y a des situations, des expériences, des sujets qui sont en situation (mais qu'est-ce que situer veut dire dans ce cas ?) d'en témoigner exemplairement. Cette exemplarité ne se réduit plus simplement à celle de l'exemple dans une série. Ce serait plutôt l'exemplarité - remarquable et remarquante - qui donne à lire de façon plus fulgurante, intense, voire traumatique, la vérité d'une nécessité universelle. La structure apparaît dans l'expérience de la blessure, de l'offense, de la vengeance, de la lésion. »
} 
cette éthique qui est aussi mise en pratique solidaire liant étroitement observation, dialogue et réflexivité. Ce credo anime la vie de Tillion en amont et en aval de sa déportation à Ravensbrück en un "double apprentissage " (Tillion 2009) qui allie l'étude et l'expérience sans les dissocier ni les hiérarchiser quant à leur apport commun de connaissances. Ce credo traverse de manière performative l'œuvre collective du Verfügbar aux Enfers (Tillion 2005 $\mathrm{a}^{18}$ ). Cette opérette-revue lue 70 ans après son écriture (dé)montre un apprentissage de l'adversité au présent, un apprentissage de la distanciation face à l'adversité du présent de l'épreuve. Parce qu'elle permet de ne pas être "écrasé » (Todorov 2000, p. 341), de ne pas demeurer sidéré, la démarche pour comprendre est salvatrice en soi, elle est apprentissage. En partage, cette action est une autre manière de se soucier de l'autre ${ }^{19}$ et de prendre soin de lui, d'apprendre de lui sur soi. À l'échelle groupale, cette entreprise a tout à voir avec la solidarité si l'on entend par solidarité le rapprochement interindividuel, le tissage des relations ${ }^{20}$ : le solidus, étymologiquement, " entendu comme fait d'un même matériau, d'un seul tenant » (Gosseries 2006, p. 1115). C'est cette leçon d'apprentissage qui est de mise en paroles et en actes dans le Verfügbar aux Enfers.

Germaine Tillion, dans son troisième Ravensbrück paru en 1988, établit, en étendant son constat aux soldats anglais, américains ou russes qui entrèrent les premiers à Bergen-Belsen, Dachau ou Auschwitz et " participèrent aussitôt aux sentiments intenses des victimes ", que " les témoignages directs sur la déportation excluent la neutralité et l'excluent même au maximum, car un des caractères du régime concentrationnaire fut de n'avoir jamais été vu par un "spectateur" indifférent » (Tillion 1988, p. 304-306). Paradoxalement, c'est bien cette singularité de la non-neutralité dans l'univers concentrationnaire que l'opérette-revue retourne en la diffractant dans : une mise en scène de la distanciation d'un intérieur démultiplié (intérieur du camp, intérieur de personnages de diverses conditions, intérieur de voix polyphoniques (for) intérieur de l'ironie, de l'humour noir et de l'autodérision) ; la fabrication d'un " "spectateur" indifférent» (conférencier bavard qui objective le Verfügbar" ${ }^{21}$ pour son étude, Verfügbar silencieux tandis qu'il est assujetti aux traitements les plus cruels). L'opérette-revue redonne à lire, à voir et à écouter la mémoire musicale d'un passé chéri désormais disparu, elle le réifie dans ce contexte d'extrême violence, par bribes, par détournements successifs, par écarts et rebonds, par déformations parodiques et glissements sémantiques, par manipulations et déplacements des airs, des paroles, des gestes. L'évocation nostalgique du passé se brise sur l'implacable horreur du présent

18 Les références à ce texte seront toutes indiquées directement entre parenthèses après la mention (LVE, p.).

19 Todorov (1991, p. 82), oppose ce souci de l'autre, ce « souci pour quelqu'un ", au « sentiment de solidarité qui peut se manifester en toutes sortes de situations » et qui finit par impliquer l'exclusion des autres puisqu'il privilégie le groupe. Nous verrons plus loin qu'en dépliant le lien interindividuel dans le groupe on peut retrouver ce souci de l'autre qui dépasse la sphère groupale.

20 Remarquons dans tous les textes de Tillion le nombre important d'occurrences du verbe « tisser ».

21 Pour une définition des prisonnières "Verfügbar », voir l'article de Marie-Hélène Benoit-Otis et Philippe Despoix dans le présent numéro. 
et s'achève en effet sur le récit du cas de Sympathie, un Verfügbar que rien - pas même les pires sévices - ou presque ne fait plus réagir. Le lecteur du Verfügbar aux Enfers demeure interdit par le choc que lui inflige une " métaphysique » du bobard délivrée en clausule. Peut-être l'est-il aussi, car brutalement revenu, au terme de l'opéretterevue, au réalisme des conditions d'écriture et de la possible ou impossible survie des déportées qui en sont les auteures :

Bérangère me cachait dans une caisse, a raconté Germaine Tillion, et là j'ai écrit une opérette en y introduisant des chansons dont nous inventions les paroles au fur et à mesure en allant et en revenant du travail. Les SS aimaient ça, car cela faisait partie de leur système. Nous marchions au pas en chantant des vers dans lesquels nous les tournions en ridicule, mais aucun ne comprenait le français ! C'était au fond une blague collective (Mestre et Moro 2004, p. 20).

\section{L'ÉPREUVE PARTAGÉE}

Les ressorts du comique percent le fonctionnement de la terrifiante machine concentrationnaire. Entre ces deux personnages - le Naturaliste et Sympathie - qui de l'intérieur du camp font montre d'indifférence, mais pour des raisons antithétiques, les protagonistes décrivent et expliquent avec une lucidité effrayante ponctuée d'autodérision ce qui les anéantit littéralement. Aussi leur univers se trouve-t-il paradoxalement déconstruit. Mais si une pulsion de vie en est irrémédiablement propulsée en emblème d'espérance, c'est souvent essentiellement pour sa dimension d'épreuve partagée. Citons en exemple l'acte II qui s'ouvre sur un ballet avec des " girls" chantant et dansant tout en " tuyautant " : un des travaux composant le terrassement. Après avoir évoqué diverses activités tout autant extrêmement pénibles toujours liées au terrassement (rouleau, brouettes) et à la contrainte (la terreur les chiens), les protagonistes expliquent au Naturaliste ce qu'est le "Planirung » (sic) et entreprennent de montrer ce qu'est le travail de la route, le travail de terrassière. La chanson "La route est longue, longue, longue " est d'abord entonnée par Dédé de Paris puis est reprise par le Chœur. Elle fonctionne sur un double discours, une ironie sur la longueur (et la dureté et la durée) de cette route : plus la route est longue, plus le travail est pénible et risque d'achever les terrassières, plus la route est longue plus le maintien en vie des terrassières est assuré, plus la route est longue plus leur résistance et leur sabotage est effectif, plus la route est longue plus celles qui remplaceront les terrassières auront aussi à faire et à faire durer la route. Deux autres chansons sur la route suivront. Dans l'interprétation de Dédé de Paris ( $L V E$, p. 132), on notera le passage d'une adresse au " tu » (au Chœur, au collectif comme un « tu », " Elle chante tournée vers le Chœur ", précise la didascalie) vers une description "tournée vers le public » (précise la didascalie) qui désignera les protagonistes par " elles ». Trois temps se succèdent : un impératif (l'assignation au travail forcé), un futur simple (la fatalité implacable des tâches obligatoires) et un présent de description qui montre la pénibilité du travail, mais aussi son détournement à des fins de résistance et de sabotage. En faisant semblant de travailler, les terrassières rendent la route encore plus longue « car elles remuent beaucoup la terre, / Mais surtout sans la déplacer ». 
Le Chœur reprend la chanson ( $L V E$, p. 134) en déplaçant à son tour le sujet vers le " nous » : le collectif alors s'exprime au passé composé ("Mais nous n'lavons guère allongée ", " Nous n'avons cessé de penser ») en évoquant le sabotage par l'action collective : une volonté groupale au présent ("Mais nous voulons la faire durer ») qui laisse entrevoir la perspective inévitablement funeste de ce travail. À un moment les terrassières seront remplacées (trop épuisées, elles seront vouées à la mort), mais, acte de résistance collective, elles laisseront beaucoup de labeurs aux prochaines terrassières.

Le sens global du texte source («La route est longue » écrit vers 1938 par Francine Cockenpot $^{22}$ ) est métaphore du chemin de l'existence que chacun doit emprunter : il égrène les obstacles à franchir, vante l'abnégation à revêtir, fait miroiter l'issue de cette épreuve longue, mais combien formatrice et compensatrice de l'effort consenti en un lumineux accès à la providence. A contrario, le chant de Dédé de Paris, et encore plus celui du Chœur, ne sont imprégnés d'aucun espoir. Pire, le chant qui libère dans la chanson scoute est le chant aliénant et performatif du travail et du sabotage même, un chant qui décrit et décrie un travail comme une épreuve, une route, sans aucune fin. Le détournement ironique est au centre de cette longueur, de cette dureté et de cette durée : la route sans fin devient « la fin », moins vite elle se terminera plus l'action de résistance aura été efficace. Pas d'espoir dans le chant du Verfügbar sur cette route si ce n'est dans l'action collective, ce «nous » qui, parce que collectif, résiste en poussant l'absurdité du travail à son extrême : faire durer la dureté et la longueur de cette route. Du texte source au texte du Verfügbar aux Enfers, l'adresse au " tu » est largement dépassée, elle se distancie aux " elles » pour ensuite englober le collectif et c'est à cette condition qu'une finalité (désespérée) est trouvée. D'un côté, le chant de scout à la gloire du chemin de l'existence est semé d'embuches, mais le sujet y triomphe de l'adversité en trouvant la lumière. De l'autre, le chant du Verfügbar est tragique, car sans issue si ce n'est la possibilité dérisoire du choix du sabotage quasi symbolique (le "Planirung " fonctionne de toute façon très bien tout comme l'énorme machine à broyer la résistance physique de ces femmes). Entre les deux chants, l'écart est celui du cynisme et du désespoir.

Selon Berenice Fischer et Joan Tronto, le care est

une activité générique qui comprend tout ce que nous faisons pour maintenir, perpétuer et réparer notre "monde ", en sorte que nous puissions y vivre aussi bien que possible. Ce monde comprend nos corps, nous-mêmes et notre environnement, tous éléments que nous cherchons à relier en un réseau complexe, en soutien à la vie (Fischer et Tronto 1991, p. 40).

Dans le Verfügbar aux Enfers, l'ironie travaille au point de retourner la fonction du care de "réparer notre monde " et de la déplacer : "saboter notre monde [du camp] en sorte que nous puissions y vivre aussi [longtemps] que possible » (ibid.).

22 Pour un accès aux paroles de cette chanson scout : https://www.youtube.com/ watch?v=L9q4WCV1Z0I (consulté le 15 janvier 2016). 
LA « MÉTAPHYSIQUE » DU BOBARD

L'opérette-revue fabrique ce " "spectateur" indifférent " : non seulement avec le personnage (temporaire) du Naturaliste investi de sa docte mission-obsession d'observateur-donneur de leçon sur la définition du Verfügbar, mais surtout, de manière redoublée, avec le personnage (final) de Sympathie qui figure l'indifférence personnifiée poussée à son paroxysme puisque ce qui l'indiffère est son propre sort. Le dernier mot sur Sympathie ( $L V E$, p. 208), dernier mot de la composition collective, quant à lui, poursuit sur le mode ironique institué dans l'ensemble de l'opéretterevue, mais déploie de manière plus complexe une antithèse composée d'une série d'oxymorons. La synecdoque ${ }^{23}$ de la sympathie se déroule par hypotypose ${ }^{24}$ rythmée $^{2}$ des anaphores "On lui a fait » et "Ça lui a rien fait ", avec force de détails sur la spécificité des soins apportés : à la lettre, la sympathie est appliquée à Sympathie, cette attention à l'autre " consolatrice des maux physiques et moraux " (Grouchy [1764-1822]1993, p. 26), cette "disposition que nous avons à sentir de manière semblable à celle d'autrui » (ibid., p. 39). Alors, tous les soins dignes du camp et de sa condition de Verfügbar lui sont prodigués sans la faire sortir de son indifférence (ou lassitude extrême), ou à peine : avertissement, coup de bâton, travail forcé, coups de pied au derrière, coups de bottes dans le crâne, attaque par le chien féroce démuselé, enfermement sans nourriture dans une cave...

Antonomase $^{25}$, le nom de Sympathie est le nom propre qui dit aussi le nom commun, il est le produit du littéral, une littérarité de la langue propre au camp de concentration, « liée à la dimension performative du langage nazi, comme le souligne Bernard-Nouraud (2013, p. 67) : dans les camps, les mots en quelque sorte s'incarnaient, leur signification avait un poids, au sens littéral ». Aussi le terme " sympathie » emprunté au latin sympathia (" accord, affinité naturelle »), lui-même emprunt au grec sumpatheia, signifie " participation à la souffrance d'autrui ». Le mot est dérivé de l'adjectif sunpathès « qui prend part à la douleur d'autrui » (Rey $[1992,1998] 2004$, p. 3720). La " sympathie » dans ce cas est littéralement " participation à la souffrance » de Sympathie, " on lui a fait " subir une série de sévices, " on " (impersonnel comme l'ordre donné et exécuté) " prend part à la douleur » de Sympathie en la lui infligeant, directement. Pourtant, Sympathie, figure de la cachectique dans sa solitude et son épuisement total, d'abord psychologique, n'est pas indifférente à tout : seul le bobard parvient à la sortir de son état.

\footnotetext{
23 Nous retiendrons pour ces tropes et figures les définitions de Pierre Fontanier ([1821-1830]1977]). La synecdoque " comprend deux objets sous le nom d'un seul, ou énonce un objet au lieu d'un autre qui, se trouvant avec celui-là dans le rapport du tout à la partie, ou de la partie au tout, y tient par une intime connexion physique ou métaphysique » (ibid., p. 261-262).

24 «L'hypotypose peint les choses d'une manière si vive et si énergique, qu'elle les met en quelque sorte sous les yeux, et fait d'un récit ou d'une description, une image, un tableau, ou même une scène vivante " (ibid., p. 390).

25 L'antonomase est «l'emploi d'un nom pour un autre, mais d'un nom propre pour un nom commun, ou d'un nom commun pour un nom propre [...]» (ibid., p. 261).
} 
Alors on lui a fait le Bobard (pas le gros, le petit). Le petit a dit : « Il faut lui faire le Débarquement. » On lui a fait le Débarquement. Ça lui a rien fait. Elle en a tant vu tu penses ! Ça l'a même fait rigoler. Alors on lui a fait le Bobard (pas le petit, le gros). Le gros a dit : « Il faut lui faire 1'Armistice. » On lui a fait l'Armistice. Ça lui a enlevé la fatigue comme ave la main... (LVE, p. 216)

Sympathie est-elle revenue à la vie grâce au bobard ? Ou la fatigue disparue est-elle seulement l'euphémisme de la folie, voire de la mort? Comme l'histoire, la sociologie et la littérature des camps l'établiront plus tard, le mensonge est au cœur du système concentrationnaire et de l'entreprise d'extermination. Ce qui vient à bout de la fatigue extrême de Sympathie et la réveille, ce ne sont pas les coups et la douleur, mais des mots. C'est le plus gros des mensonges, celui qui serait encore plus difficile à croire, qui parvient à la sortir de son indifférence. Ce retour à la réalité par la lucidité de ce que dit le bobard illustre la violence infligée à la désespérée : elle rit de l'improbable, mais lorsque les mots d'un mensonge encore plus gros, impossible, sont prononcés, elle en revient littéralement. Alors que les bobards « ça fait trop mal après ", dit Lulu de Colmar (ibid.), le fin mot sur la conséquence du bobard une fois son effet passé sur ceux qui véritablement veulent y croire demeure un suspens, une rupture : "Pour les vrais bobardiers, il n'y a pas d'après. En juin on leur dit que Paris est libéré : ils sont contents. En juillet on leur dit, de source sûre, que Paris va être libéré. Ils sont encore contents " explique Havas (ibid.). Cet état de contentement (béat, crédule, suspect, désespéré ?) semble de l'ordre du refus du futur tout autant que du présent, c'est une sorte d'état d'oubli qui les maintiendrait dans un temps hors le présent, le passé et le futur, hors leur condition dans le camp... Pour Sympathie, la sympathie sur le mode ironique serait, afin de la toucher au plus profond d'elle-même, de lui " faire le bobard ». La sympathie, sur un mode non ironique, est la sollicitude pour l'autre : pour Sympathie, la sympathie serait de prononcer les mots qu'elle attend quand bien même ces mots diraient l'impossible, le plus gros des bobards, l'absurde. Dans le camp, la " métaphysique » du bobard fonctionne de manière " littérale ", soit de manière « rigoureusement conforme à la vérité, à la réalité » (Rey [1992, 1998]2004, p. 2040) : elle est " un univers c'est-à-dire une métaphysique et une attitude d'esprit » (Camus 1942, p. 24) qui correspond diamétralement à ce que susciterait l'absurde tragique de la situation.

\section{SOLIDARITÉ ET CARE TOUT ENSEMBLE}

À partir de cette évocation de Sympathie peut s'opérer une (re)lecture fructueuse de ce qui fonde l'extraordinaire singularité de Germaine Tillion. Car, en de nombreux points troublants, de manière remarquable, Sophie de Grouchy (Marquise de Condorcet) et ses Lettres sur la Sympathie ne cessent de correspondre avec Germaine Tillion, de correspondre à Germaine Tillion. En fait, en lisant Les lettres sur la sympathie 
adressées à Condorcet ${ }^{26}$ emprisonné (Grouchy [1798]1993) par celle qui a traduit la Théorie des sentiments moraux, " une anthropologie de la sympathie qui assure une sorte d'harmonie naturelle préétablie entre les individus humains » (Gusdorf 1978, p. 333) développée par Adam Smith (1759), on retrouve de nombreuses préoccupations et principes chers à Germaine Tillion. Ces idées communes aux deux femmes se formulent dans des termes étonnamment proches et demeurent singulières dans l'histoire des idées à plus de deux siècles d'intervalle. D'une part, le commentaire sur Smith de Sophie de Condorcet se base sur des éléments fondés sur la prise en compte de la subjectivité et de la notion d'égalité entre les êtres quelles que soient leurs différences : savoir de l'expérience, imagination, impératif de l'instruction, valeur du rire, etc. D'autre part, ce qui émane de ces correspondances (de Sophie de Grouchy à Condorcet, des idées de Sophie de Grouchy à celles de Germaine Tillion à ce qui circule dans Le Verfügbar aux Enfers) est une réflexion éminemment contemporaine sur la morale et l'engagement, sur la solidarité et le care, sur la place de l'individu dans « notre monde » et son mode d'action pour le « réparer». Il en appert que la solidarité et le care ne devraient pas se hiérarchiser, s'opposer ni s'exclure dans la mesure où ils alterneraient comme phénomènes tout en coprésence, voire complémentaires : dans le contexte extrême du camp de concentration, ainsi que le représente et le donne à comprendre le Verfügbar aux Enfers, le " solidus " prime pour le groupe quand entre détenues le souci de l'autre est omniprésent.

\section{L'IRONIQUE ET DOUBLE SUSPENSION DE LA LITTÉRATURE}

Dans cet hapax que figure Le Verfügbar aux Enfers au milieu de l'hétérogénéité des objets artistiques qui proviennent des camps de concentration nazis - et même si l'opérette-revue ne fut jamais jouée à Ravensbrück ${ }^{27}$-, on pourrait reconnaître ce type d'œuvres « qui furent chantées un jour devant les crématoires d'Auschwitz » que Catherine Coquio oppose " aux valeurs des œuvres en tant que telles dans l'illusoire panthéon de l'art » (Coquio 2015, p. 18). Car dans l'opérette-revue, il semble bien " que quelque chose s'affirme pour prendre acte jusqu'au bout de l'historicité de l'art, responsable de son ironique suspension. Prendre acte de l'histoire, c'est recueillir ensemble la "liberté" et le "sarcasme", et cette liberté devenue sarcasme fait trembler tout l'édifice de l'art» (ibid.). Avec Le Verfügbar aux Enfers, œuvre au " second degré » (Gérard Genette) ou de « la seconde main » (Antoine Compagnon) tant y dominent l'intertextualité $^{28}$ (interdiscursivité et hypertextualité), la littérature est mise en suspens

26 Voir la présentation de Jean-Paul de Lagrave (Grouchy [1798]1993, p. 5-36) qui qualifie ces lettres de " plus petit traité du bonheur publié au $18^{\mathrm{e}}$ siècle ".

27 Ni ailleurs avant sa mise en scène au Théâtre du Châtelet en 2007.

28 Pour Genette ([1982]1992, p. 8), l'intertextualité, qui constitue l'une des cinq modalités de relations qui agissent dans la transtextualité, se définit comme « une relation de coprésence entre deux ou plusieurs textes, c'est-à-dire, eidétiquement et le plus souvent, par la présence d'un texte dans un autre. » Nous retiendrons que le théoricien " distingue transformation (parodie, travestissement, transposition) et imitation (pastiche, charge, forgerie [= le faux]), selon que la relation hypertextuelle est "ludique", "satirique" ou "sérieuse" " (Charaudeau et Maingueneau 2002, p. 298). Ces catégories genettiennes, si elles sont parfois 
deux fois. Et de façon à chaque fois pourrait-on dire effective. La littérature est mise en suspens une première fois, dans le texte, de manière métaphorique ou littérale, car elle travaille le présent, et met en jeu le suspens même : "le présent par quoi l'événement s'inscrit dans la littérature à l'état de question »(Coquio 2015, p. 12). La littérature est mise en suspens une seconde fois, car l'œuvre est empêchée - comme la mémoire empêchée décrite par Paul Ricœur ([2000]2003, p. 82-96). Durant près de 40 ans, elle est remisée, tue, mise au silence avant d'être publiée, publicisée, connue de tous dans les années 2000. Germaine Tillion, au sortir de l'épreuve concentrationnaire, a mis en attente Le Verfügbar aux Enfers, elle n'en a finalement exhumé quelques extraits que dans son troisième ouvrage consacré à Ravensbrück.

Le suspens qui travaille, constitue, définit l'écriture même de l'opérette-revue est celui que conçoit Catherine Coquio lorsqu'elle exemplifie la démarche d'écriture de l'écrivain Imre Kertész : " une mise en crise ou en doute de la "littérature", de sa pertinence, de son contenu, de son héritage. Cette mise en doute, qui est aussi condition de sa poursuite, ou de sa relance, lui est désormais constitutive » (Coquio 2015, p. 12). Et lorsque Coquio prend la mesure de l'impact de cette œuvre de Kertész, on peut par analogie appliquer ces propos à l'œuvre collective du Verfügbar aux Enfers :

La littérature ne peut plus officier sans questionner radicalement son office, sans concevoir son illégitimité ou plutôt sa dérision, alors même qu'elle s'était vue et se voit confirmée dans sa nécessité de fait, vitale, et durable, comme le dit puissamment cette œuvre sans public [si ce n'est le public potentiel premier du camp pour Le Verfügbar aux Enfers] avant d'être internationalement consacrée (ibid., p. 13).

L'opérette-revue, cette "blague collective ", " littérature en suspens ", est une " littérature à l'envers, mais aussi au carré » (ibid.) puisque la forme comme le contenu est ironique et que s'y livre un rapport de jeu entre eux (sans compter le jeu proposé de la (re)présentation). Ce rapport à la forme et au contenu, littéraire, est constitutif de l'œuvre par laquelle le collectif qui en est l'auteur a compris, sous l'impulsion de Germaine Tillion, « qu'il fallait reprendre la vie » qu'on leur retirait dans le camp " au monstrueux Moloch qu'on appelle 1'histoire » (ibid., p. 17). Reprendre la vie en jouant à la recréer, c'est l'un des enseignements, l'un des apprentissages que livre l'opérette-revue ; comme une réponse possible à la tragique question de l'utilité de la connaissance des survivants des camps de la mort pour qui cette " expérience hors de la vie ${ }^{29}$ »(Delbo 1966) a pour principale aporie l'impossibilité plus intime d'être

aisées à retrouver dans les transformations opérées sur les hypotextes, s'avèrent quelques fois peu évidentes puisqu'il arrive que dans ce procédé de la transformation du texte source, les imitations et les transformations mêlent plusieurs de leurs variantes.

29 Il serait intéressant de mener une réflexion qui ferait dialoguer cette question de « la connaissance » (Tillion) avec celle « des connaissances » (Delbo), en partant précisément de l'assertion de Delbo : «On a acquis des connaissances, c'est vrai [...] mais des connaissances qui ne peuvent pas servir, parce que c'est une expérience hors de la vie » (Charlotte Delbo [1966] citée dans Caron et Marquart 2011, p. 21). 
verbalisée et transmise. Écriture exercée cachée dans une caisse, action tout à la fois dérisoire et fondamentale, l'opérette-revue est aussi une des étapes cruciales d'apprentissage de la vie chez Germaine Tillion. Elle est bien un élément de cette « mécanique d'érudition qui ressemble aux notes d'une partition musicale » (Tillion 2009, p. 49) éminemment liée à son expérience qui est la "gamme sonore sans laquelle la partition restera morte " (ibid.).

Exemplaire est donc cette composition à plus d'un titre : de l'être à son parcours, de l'œuvre à son éthique. L'expérience, comme la mémoire, est du passé. L'action, comme l'histoire, se fabrique au présent. La connaissance émane de l'une et sert l'autre, et réciproquement. La solidarité est quant à elle affaire de choix, d'engagement exemplaire, mais surtout affaire de tissage, de tissage de relations, affaire d'individu et de groupe. La part reconnue à la subjectivité a pour corollaire l'humilité, la modestie, la générosité et l'humour qui sont autant de traits constitutifs du personnage de Germaine Tillion. Que vaut l'expérience si elle ne peut être utile à la connaissance ? Quelle valeur revêt la connaissance si elle ne peut permettre d'agir? Une connaissance qui ne peut être communiquée, comprise, partagée avec autrui en est-elle encore une ? Si l'entraide est exclue, que valent expérience, connaissance et action face à l'adversité ? On l'aura saisi, c'est moins à une rhétorique de la mesure - vaine sans doute - de la valeur d'un savoir à l'aune de son utilisation pratique qu'à partir de ces questionnements éthiques - et épineux - qu'invite à réfléchir le parcours de la vieille dame.

\section{BIBLIOGRAPHIE}

Bernard-Nouraud, Paul (2013), Figurer l'autre. Essai sur la figure du "musulman " dans les camps de concentration nazis, Paris, Kimé.

Bouchet, Francis, et Michel Anthonioz (1974), Germaine Tillion. Deux fils qui se croisent et La république des cousins, Paris, ORTF, 54 et 44 min.

Bromberger, Christian (2009), "Germaine Tillion (1907-2008) ", L'Homme, 2009/1, n 189, p. 1122. Disponible en ligne : https://www.cairn.info/revue-1-homme-2009-1.htm, consulté le 29 février 2016.

Bromberger, Christian, et Tzvetan Todorov (2002), Germaine Tillion. Une ethnologue dans le siècle, Arles, Actes Sud.

Camus, Albert (1942), Le mythe de Sisyphe. Essai sur l'absurde, Paris, Gallimard.

Caron, David et, Marquart, Sharon (dir.) (2011), Les revenantes. Charlotte Delbo : la voix d'une communauté à jamais déportée, Toulouse, Presses universitaires du Mirail.

Charaudeau, Patrick, et Maingueneau, Dominique (dir.) (2002), Dictionnaire d'analyse du discours, Paris, Seuil.

Chapsal, Madeleine (1966), «Rien que des femmes. Entretien avec Charlotte Delbo », L'Express, $\mathrm{n}^{\circ} 765$ (14-20 février), p. 74-76.

Chiantaretto, Jean-François (2005), Le témoin interne. Trouver en soi la force de résister, Paris, Flammarion/Aubier.

Coquio, Catherine (2015), La Littérature en suspens. Écritures de la Shoah : le témoignage et les ceuvres, Paris, L'Arachnéen. 
Derrida, Jacques (1996), Le monolinguisme de l'autre ou la prothèse de l'origine, Paris, Galilée. Disponible en ligne : http://xn--entre-1-fwa.net/wp-content/uploads/2014/07/Derrida-Le-monolinguismede-lautre1.pdf, consulté le 29 février 2016.

Fischer, Berenice, et Joan Tronto (1991), « Toward a Feminist Theory of Care », dans Emily K. Abel et Margaret K. Nelson (dir.), Circles of Care. Work and Identity in Women's Lives, Albany, NY, State University of New York Press, p. 35-62.

Fontanier, Pierre ([1821-1830]1977), Les figures du discours, introduction par Gérard Genette, Paris, Flammarion.

Forget, Nelly (2013), « L'engagement de Germaine Tillion dans le service des Centres sociaux en Algérie ", dans Armelle Mahon et Gwendal Simon (dir.), L'engagement à travers la vie de Germaine Tillion, Paris, Riveneuve éditions, p. 37-60.

Gastaut, Yvan (2000), L'immigration et l'opinion en France sous la ve République, Paris, Seuil.

Genette, Gérard ([1982]1992), Palimpsestes. La littérature au second degré, Paris, Seuil.

Gosseries, Axel (2006), "Solidarité », dans Sylvie Mesure et Patrick Savidan (dir.), Le dictionnaire des sciences humaines, Paris, PUF, p. 1114-1116.

Grouchy, Sophie de, marquise de Condorcet ([1764-1822]1993), Lettres sur la sympathie; suivies des Lettres d'amour à Maillia Garat, textes revus, présentés et annotés par Jean-Paul de Lagrave, Montréal, Presses de 1'Université du Québec.

Gusdorf, Georges (1978), Les sciences humaines et la pensée occidentale, " Tome VIII. La conscience révolutionnaire. Les idéologues », Paris, Payot.

Kelle, Michel ([2013]2014), "Germaine Tillion (1907-2008). Ethnologue, résistante et militante », dans Michel Kelle, Cinq figures de l'émancipation algérienne. Des modèles pour un renouveau des rapports franco-algériens?, Alger, Casbah Éditions, p. 15-80.

Mestre, Claire, et Marie-Rose Moro (2004), « Une pensée et un engagement. Entretien avec Germaine Tillion ", L'Autre, vol. 5, n 1, p. 7-28.

Ould Aoudia, Jean-Philippe (2013), «Les centres sociaux et la guerre d'Algérie », dans Armelle Mahon et Gwendal Simon (dir.), L'engagement à travers la vie de Germaine Tillion, Paris, Riveneuve éditions, p. 49-60.

Reid, Daniel (2004), «From Ravensbrück to Algiers and Noisy-le-Grand. Dialogues with Deportation», French Politics, Society \& Culture, vol. 22, no 3 (automne), p. 1-24.

Reid, Daniel (2007), "De Ravensbrück à Alger ", dans Tzvetan Todorov (dir.), Le siècle de Germaine Tillion, Paris, Seuil, p. 149-171.

Rey, Alain (dir.) ([1992, 1998]2004), Le Robert. Dictionnaire historique de la langue française, Paris, Dictionnaires Le Robert.

Ricour, Paul ([2000]2003), La mémoire, l'histoire, l'oubli, Paris, Seuil.

Rothberg, Michael (2009), Multidirectional Memory. Remembering the Holocaust in the Age of Decolonization, Standford, Standford University Press.

Saadi, Yacef ([1984]1997), La bataille d'Alger, « Tome II. L'affrontement », Alger, Casbah Éditions.

Smith, Adam ([1759]1976), The Theory of Moral Sentiments, Oxford, Oxford University Press.

Tillion, Germaine (1957), Algérie 1957, Paris, Les Éditions de Minuit.

Tillion, Germaine (1960), L'Afrique bascule vers l'avenir, l'Algérie en 1957 et autres textes, Paris, Les Éditions de Minuit.

Tillion, Germaine (1966), Le harem et les cousins, Paris, Seuil.

Tillion, Germaine (1973), Ravensbrück, Paris, Seuil.

Tillion, Germaine (1988), Ravensbrück (suivi de Les exterminations par gaz à Hartheim, Mauthausen et Gusen par Anise Postel-Vinay et Pierre-Serge Choumoff), Paris, Seuil. 
Tillion, Germaine (1998), «En souvenir de David Rousset », Voix et Visages, n 258, p. 3.

Tillion, Germaine (2000), Il était une fois l'ethnographie, Paris, Seuil.

Tillion, Germaine (2001), À la recherche du vrai et du juste. Propos rompus avec le siècle, textes réunis et présentés par Tzvetan Todorov, Paris, Seuil.

Tillion, Germaine (2005a), Le Verfügbar aux Enfers. Une opérette à Ravensbrück, présentation de Tzvetan Todorov et Claire Andrieu, Paris, La Martinière.

Tillion, Germaine (2005b), Les ennemis complémentaires, Guerre d'Algérie, Préface de Jean Daniel, Paris, Éditions Tirésias.

Tillion, Germaine (2009), Fragments de vie, textes rassemblés et présentés par Tzvetan Todorov, Paris, Seuil.

Tillion, Germaine, et al. (1946), Ravensbrück, Neuchâtel, Éditions de la Baconnière.

Todorov, Tzvetan (1991), Face à l'extrême, Paris, Seuil.

Todorov, Tzvetan (1995), Les abus de la mémoire, Paris, Arléa.

Todorov, Tzvetan (2000), Mémoire du mal, tentation du bien, Paris, Robert Laffont.

Todorov, Tzvetan (dir.) (2007), Le siècle de Germaine Tillion, Paris, Seuil.

Vautier, René (2013), "Dialogue inédit [avec Germaine Tillion] ", dans Armelle Mahon et Gwendal Simon (dir.), L'engagement à travers la vie de Germaine Tillion, Paris, Riveneuve éditions, p. 191-198.

Vidal-Naquet, Pierre (1972), La torture dans la République. Essai d'histoire et de politique contemporaine (1954-1962), Paris, Les Éditions de Minuit.

Vidal-Naquet, Pierre (1995), « Réflexions sur trois Ravensbrück », dans Pierre Vidal-Naquet, Les Juifs, la mémoire, le présent, "Tome iII. Réflexions sur le génocide », Paris, La Découverte, p. 195-208.

Vidal-Naquet, Pierre (2007), "Réflexion sur les trois Ravensbrück », dans Tzvetan Todorov (dir.), Le siècle de Germaine Tillion, Paris, Seuil, p. 315-148.

Wood, Nancy (2003), Germaine Tillion, une femme-mémoire, d'une Algérie à l'autre, Paris, Autrement. 